\title{
A NON SIMPLICITY CRITERION FOR FINITE GROUPS
}

\author{
NITA BRYCE
}

(Received 1 February 1967)

M. Suzuki [3] has proved the following theorem. Let $G$ be a finite group which has an involution $t$ such that $C=C_{G}(t) \cong S L(2, q)$ and $q$ odd. Then $G$ has an abelian odd order normal subgroup $A$ such that $G=C A$ and $C \cap A=\langle 1\rangle$.

We can prove the following similar result:

THEOREM. Let $G$ be a finite group of even order with the following property:

(a) $G$ has an involution $t$ such that the centralizer $C=C_{G}(t)$ is the central product of $C_{1}$ and $C_{2}$ where $C_{i} \cong S L\left(2, q_{i}\right)$ and $q_{i}$ odd, $i=1,2$. Then $G$ has an abelian odd order normal subgroup $A$ such that $G=C A$ and $C \cap A=\langle 1\rangle$.

At first we give two definitions.

Definition. A group $G$ is called the central product of two subgroups $H_{1}$ and $H_{2}$ if $G=H_{1} H_{2}$ and $\left[H_{1}, H_{2}\right]=1$, (i.e. $H_{1}$ and $H_{2}$ commute elementwise).

Definition. Let $\alpha$ be an automorphism of a group $G$. Then $\alpha$ is called fixed-point-free if and only if $\alpha$ fixes only the unit element of $G$.

In the proof of the theorem we shall use the following result.

Frattini Lemma [1]. Let $N$ be a normal subgroup of a finite group $G$, and let $K$ be a Sylow p-subgroup of $N$ for some prime $p$. Then $G=N N_{G}(K)$.

TheOREM OF GLAUberman [2]. Let $t$ be an involution contained in a Sylow 2-subgroup $S$ of a finite group $G$. It $t$ is not conjugate in $G$ to any other involution $t^{\prime} \neq t$ of $S$, then $t \in Z\left(G \bmod O_{2^{\prime}}(G)\right)$, where $O_{2^{\prime}}(G)$ is the maximal normal odd order subgroup of $G$.

Theorem of Zassenhaus [4]. If a finite group $G$ has a fixed-point-free automorphism of order 2 , then $G$ is an abelian group of odd order.

We now prove some preliminary results. The first lemma is well known.

Lemma 1. An $S_{2}$-subgroup of $S L(2, q)$, q odd, is a generalized quaternion group. 
Iемма 2. Let $Q_{i}$ be an $S_{2}$-subgroup of $C_{i}, i=1,2$. Then $Q_{1} \cap Q_{2}=\langle t\rangle$ and $Q=Q_{1} Q_{2}$ is an $S_{2}$ subgroup of $C$ and hence of $G$.

ProOf. $Q_{i}=\left\langle a_{i}, b_{i} \mid a_{i}^{2^{n_{i}-2}}=b_{i}^{2}=t, b_{i}^{-1} a_{i} b_{i}=a_{i}^{-1}\right\rangle$ is an $S_{2}$-subgroup of $C_{i}, i=1,2$. Since $t \in Q_{i}, i=1,2$, then $\langle t\rangle \subseteq Q_{1} \cap Q_{2}$. On the other hand, $Q_{1} \cap Q_{2} \subseteq C_{1} \cap C_{2}=\langle t\rangle$, so $\langle t\rangle=Q_{1} \cap Q_{2}$. By consideration of the orders of $C_{i}, Q=Q_{1} Q_{2}$ is an $S_{2}$-subgroup of $C$. Let $T$ be an $S_{2}$-subgroup of $G$ containing $Q$. Since $Q$ is an $S_{2}$-subgroup of $C$ then $T \cap C=Q$. Now $Z(T) \subseteq C=C_{G}(t)$ and so $Z(T) \subseteq Z(Q)=\langle t\rangle$. Thus $\langle t\rangle=Z(T)$ giving $T \subseteq C$ and hence $T=Q$.

LEMMA 3. Every involution $\tilde{t} \neq t$ of $C$ is of the form $\tilde{t}=x_{1} x_{2}$ where $x_{i} \in C_{i}, i=1,2$, is an element of order 4 .

Proof. Since $C=C_{1} C_{2}$, every non-central involution $\tilde{t}$ can be written as $t=x_{1} x_{2}$ where $x_{i} \in C, i=1,2$. Because $\tilde{t}^{2}=1, \tilde{t}^{2}=x_{1}^{2} x_{2}^{2}=1$ and so $x_{1}^{2}=\left(x_{2}^{2}\right)^{-1} \in C_{1} \cap C_{2}=t$. Thus either $x_{i}^{2}=1$ or $x_{i}^{2}=t$. But $x_{i}^{2} \neq 1$ since $t$ is the only involution of $C_{i}$. Hence $x_{i}$ is of order 4 .

LEMMA 4. $C$ has two conjugate classes of involutions with representatives $t$ and

$$
t_{1}=a_{1}^{2^{n_{1}-3}} a_{2}^{2^{n_{2}-3}} .
$$

Proof. From the assumptions of our theorem $\langle t\rangle=Z(C)$ and so $t$ forms a conjugate class of involutions of $C$. By Lemma 3 , every non-central involution of $C$ has the form $x_{1} x_{2}$ where $x_{i}$ is an element of order 4 in $C_{i}, i=1,2$. However, all elements of order 4 in $C_{i}$ (fixed $i$ ) are conjugate in $C_{i}$ since $C_{i} \mid\langle t\rangle \cong P S L\left(2, q_{i}\right)$ which has only one class of involutions. Hence any non-central involution in $C$ is conjugate to

$$
t_{1}=a_{1}^{2^{n_{1}-3}} a_{2}^{2^{n_{2}-3}} \text {. }
$$

LEMMA 5. The whole group $G$ has at most two conjugate classes of involutions.

Proof. This follows from Lemma 2, Lemma 4 and the theorems of Sylow.

LEMmA 6. We have $Q=C_{Q}\left(t_{1}\right)=\left\langle a_{1}, a_{2}, t_{2}\right\rangle$ and $(\widetilde{Q})^{\prime}=\left\langle a_{1}^{2}, a_{2}^{2}\right\rangle$ where $t_{2}=b_{1} b_{2}$. Also $\tilde{Q}$ is an $S_{2}$-subgroup of $C_{C}\left(t_{1}\right)$.

Proof. By a straight forward computation $C_{Q}\left(t_{1}\right)=\left\langle a_{1}, a_{2}, t_{2}\right\rangle=\tilde{Q}$, which is a non-abelian group of order

$$
2^{n_{1}+n_{2}-2} \text {. }
$$

We may write $\tilde{Q}=\left\langle t_{2}\right\rangle\left\langle a_{1}, a_{2}\right\rangle$ where $\left\langle a_{1}, a_{2}\right\rangle=H$ is abelian. Since $\left[a_{1}, t_{2}\right]=a_{1}^{-2}$ and $\left[a_{2}, t_{2}\right]=a_{2}^{-2}$, we get $K=a_{1}^{2}, a_{2}^{2} \subseteq(\widetilde{Q})^{\prime}$. Now $\unlhd H$, since 
$H$ is abelian, and $K$ remains invariant under the action of the involution $t_{2}$. Thus $K \unlhd \widetilde{Q}$. Consider $\widetilde{Q} / K$. Since $t_{2}, a_{1}, a_{2}$ all commute modulo $K$, then $K \supseteqq(\widetilde{Q})^{\prime}$. Hence $\left\langle a_{1}^{2}, a_{2}^{2}\right\rangle=K=(\widetilde{Q})^{\prime}$. Let $\widetilde{T}$ be an $S_{2}$-subgroup of $C_{C}\left(t_{1}\right)$. Suppose $\tilde{T} \supset \widetilde{Q}$. Then $\tilde{T}$ is conjugate to $Q$ and so $Z(\widetilde{T})$ is conjugate to $Z(Q)$, that is, $t_{1}$ is conjugate to $t$, a contradiction. Hence $\widetilde{T}=\widetilde{Q}$.

Lemma 7. The subgroup $\langle t\rangle$ is characteristic in $(\widetilde{Q})^{\prime}$ and hence $\langle t\rangle$ is characteristic in $C_{Q}\left(t_{1}\right)$.

Proof. We note that $K$ is abelian and consider the series

$$
\Omega^{1}(K) \supseteqq \Omega^{2}(K) \supseteqq \ldots,
$$

where $\Omega^{i}(K)$ is the subgroup of $K=(\tilde{Q})^{\prime}$ generated by all the elements $x \in K$ such that $x=y^{2^{i}}$ for some $y \in K$. Clearly, $\Omega^{i}(K) \supseteqq \Omega^{i+1}(K), i \geqq 1$. Let $\alpha$ be an automorphism of $K$ and $x \in \Omega^{i}(K)$. Then

$$
x^{\alpha}=\left(y^{2^{i}}\right)^{\alpha}=\left(y^{\alpha}\right)^{2^{i}} \in \Omega^{i}(K)
$$

for some $y \in K$ and so $\Omega^{i}(K)$ is characteristic in $K, i \geqq 1$.

Suppose $n_{1}=n_{2}=n$, then $\left|a_{1}\right|=\left|a_{2}\right|=2^{n-1}$. We have $K=\left\langle a_{1}^{2}, a_{2}^{2}\right\rangle$,

$$
\Omega^{1}(K)=\left\langle a_{1}^{2^{2}}, a_{2}^{2^{2}}\right\rangle, \ldots, \Omega^{n-3}(K)=\left\langle a_{1}^{2^{n-2}}, a_{2}^{2^{n-2}}\right\rangle=\langle t\rangle .
$$

So $\langle t\rangle$ is characteristic in $(\widetilde{Q})^{\prime}$ in this case.

Suppose $n_{1} \neq n_{2}$. Without loss of generality we may take $n_{1}>n_{2}$. Again

$$
\begin{aligned}
& \Omega^{1}(K)=\left\langle a_{1}^{2^{2}}, a_{2}^{2^{2}}\right\rangle, \quad \Omega^{2}(K)=\left\langle a_{1}^{2^{3}}, a_{2}^{2^{3}}\right\rangle, \ldots, \\
& \Omega^{n_{2}-2}(K)=\left\langle a_{1}^{2_{2}-1}, a_{2}^{2^{n_{2}-1}}\right\rangle=\left\langle a_{1}^{2^{n_{2}-1}}\right\rangle, \ldots
\end{aligned}
$$

Thus $\Omega^{n_{2}-2}(K)$ is cyclic, so $\langle t\rangle$ is characteristic in $\Omega^{n_{2}-2}(K)$ and hence $\langle t\rangle$ is characteristic in $K$.

LeMma 8. The group $G$ has precisely two conjugate classes of involutions with the representations $t$ and $t_{1}$.

Proof. Suppose $t$ is conjugate in $G$ to $t_{1}$. Then in particular,

$$
C_{G}(t)=C \cong C_{G}\left(t_{1}\right) \text {. }
$$

We know that $C_{Q}\left(t_{1}\right)=\left\langle a_{1}, a_{2}, t_{2}\right\rangle$ is an $S_{2}$-subgroup of $C_{C}\left(t_{1}\right)$, and that $\langle t\rangle$ is characteristic in $C_{Q}\left(t_{1}\right)$. Let $\tilde{T}$ be an $S_{2}$-subgroup of $C_{G}\left(t_{1}\right)$ which contains $C_{Q}\left(t_{1}\right)=\tilde{Q}$. Then $\left|T: C_{Q}\left(t_{1}\right)\right|=2$ and so $C_{Q}\left(t_{1}\right) \unlhd \tilde{T}$. Thus $\langle t\rangle \unlhd \tilde{T}$, so $\widetilde{T} \subseteq C_{G}(t)=C$, a contradiction. Thus $t$ is not conjugate in $G$ to any involution $t_{1} \neq t$ of $Q$.

PROOF OF THE THEOREM. We proceed by induction on the order of the group $G$. Denote by $O_{\mathbf{2}^{\prime}}(G)$ the maximal normal odd order subgroup of $G$. 
Suppose $O_{2^{\prime}}(G) \neq 1$. Put $\bar{G}=G / O_{2^{\prime}}(G)$. Denote by $\bar{S}$ the image in $\bar{G}$ of any subset $S$ of $G$, i.e.

$$
\bar{S}=S 0_{2^{\prime}}(G) / 0_{2^{\prime}}(G) .
$$

Let $M=0_{2^{\prime}}(G)$. Clearly, $C_{\bar{G}}(t)=C^{*} / M$, for some subgroup $C^{*}$ of $G$ containing $M$ and $t$. Write $\langle t\rangle M=N \subseteq C^{*}$. Then $N \unlhd C^{*}$ since

$$
N / M=\langle\bar{t}\rangle \unlhd C_{\bar{G}}(\bar{t})=C^{*} / M .
$$

Clearly, $\langle t\rangle$ is an $S_{2}$-subgroup of $N$. By the Frattini argument, $C^{*}=N_{C^{*}}(\langle t\rangle) N$. Since $\langle t\rangle$ is a group of order $2, N_{C^{*}}(\langle t\rangle)=C_{C^{*}}(t)$. Thus

$$
C^{*}=C_{C^{*}}(t) N=C_{C^{*}}(t)\langle t\rangle M=C_{C^{*}}(t) M .
$$

Since $C=C_{G}(t) \subseteq C^{*}$, we get $C^{*}=C M$. From the structure of $C$ we know that $O_{2^{\prime}}(C)=\langle 1\rangle$ so $C \cap M=\langle 1\rangle$. We conclude that

$$
C_{\bar{G}}(l)=C^{*} / M=C M / M \cong C .
$$

Thus the group $\vec{G}$ satisfies the condition (a) of our Theorem, and $|\vec{G}|<|G|$, so by induction the theorem is true for $\bar{G}$. But $O_{2^{\prime}}(\bar{G})=\langle\mathbf{1}\rangle$ since $\bar{G}=G / O_{2^{\prime}}(G)$, hence $\bar{G}=C_{\bar{G}}(\bar{t})$ and so $G=C O_{2^{\prime}}(G)$ and $C \cap O_{2^{\prime}}(G)=\langle 1\rangle$. Now the involution $t$ acts fixed-point-free on $O_{2^{\prime}}(G)$, so by the result of Zassenhaus [4], $O_{2^{\prime}}(G)$ is abelian. Hence our theorem is true if $O_{2^{\prime}}(G) \neq\langle\mathbf{1}\rangle$.

We may assume now that $O_{2^{\prime}}(G)=\langle 1\rangle$. But then by the theorem of Glauberman [2], $t \in Z(G)$ and so $G=C_{G}(t)=C$. The theorem is proved.

REMARK. It was kindly pointed out by the referee that this paper in fact proves the following slightly stronger result:

If $C / O_{2^{\prime}}(C)$ is isomorphic to the central product of $C_{1}$ and $C_{2}$, then $G=\mathrm{CO}_{2^{\prime}}(G)$ and $C \cap O_{2^{\prime}}(G)=O_{2^{\prime}}(C)$.

\section{Acknowledgement}

The author is indebted to Professor Z. Janko who suggested and supervised this research, as part of the requirements for the degree of Master of Science.

\section{References}

[1] R. Baer, 'Classes of finite groups and their properties', Illinois J. of Math. 1 (1957), 115-187.

[2] G. Glauberman, 'Central elements in core-free groups'. J. of Algebra 4 (1966), 403-420.

[3] M. Suzuki, 'On finite groups with cyclic Sylow subgroups for all odd primes', Amer. J. Math. 77 (1955), 657-691.

[4] H. Zassenhaus, 'Kennzeichnung endlicher linearen Gruppen als Permutationsgruppen', Abh. Hamburg Math. Sem. 11 (1936), 17-40.

Monash University, Clayton, Victoria 